\title{
Does Neuroplasticity Support the Hypothesis of Multiple Realizability?
}

\author{
Amber Maimon and Meir Hemmo ${ }^{1}$
}

\begin{abstract}
It is commonly maintained that neuroplastic mechanisms in the brain provide empirical support for the hypothesis of multiple realizability. We show in various case studies that neuroplasticity stems from preexisting mechanisms and processes inherent in the neural (or biochemical) structure of the brain. We argue that not only does neuroplasticity fail to provide empirical evidence of multiple realization, its inability to do so strengthens the mind-body identity theory. Finally, we argue that a recently proposed identity theory called Flat Physicalism can be enlisted to explain the current state of the mind-body problem more adequately.
\end{abstract}

\footnotetext{
${ }^{1}$ Philosophy Department, University of Haifa, 199 Aba Khoushy Ave. Mount Carmel, Haifa, 3498838, Israel
} 
1. Introduction. Many of today's theories regarding the mind-body problem attempt to cope with multiple realization, including all forms of nonreductive physicalism, as well as the functionalist theory of mind. By and large, the instantiation of this argument revolutionized the previously bipolar field of addressing the mind-body question via reductive physicalist vs. nonreductive dualist approaches, as well as (arguably) the scientific investigation of mind and cognition. The idea that a single mental kind (or type) can be realized by distinct physical kinds (within the brain or among species; where by 'physical kind' we mean kinds that appear in physics, including high-level physics, chemistry as well as biology). This idea knocked a blow to the reductionist identity theory (see, e.g., Putnam 1975, 1967; Fodor 1974; and also, Lewis 1966; Davidson 1970; for a recent review of the mind-brain identity theory, see Smart 2017). In contemporary literature, many philosophers share the view that "If there is now a received view among philosophers of mind and philosophers of biology about reductionism, it is that reductionism is mistaken. And if there is now a received view as to why reductionism is wrong, it is the multiple realizability argument" (Sober 1999, 542).

Putnam, though he never uttered the phrase, was the founding father of the multiple realizability hypothesis. His formulation of the hypothesis was so influential that: "Seemingly overnight Putnam's multiple-realization argument convinced philosophers that the mind-brain identity theory is untenable" (Polger and Shapiro 2016, 12). The multiple realizability hypothesis remains the most popular argument against identity theory to this day.

With relation to neuroscience, one of the most well-known families of cases thought to support the multiple realizability (or realization) hypothesis is the phenomena of neuroplasticity, as expressed by Endicott: "It is now commonplace to observe that mental properties can be "multiply realized" by an indefinite number of physically dissimilar systems, and in such a way 
as to preclude any straightforward identification with physical properties" (Endicott 1993, 303). Furthermore, "the most interesting problem for the NRS [narrow reductive strategy] arises not from outside human psychology, but from the way our own biological species happens to instantiate mental properties. Here I refer to the well-known problem created by the plasticity of the brain" (Endicott 1993, 312).

In this paper, we argue that neuroplasticity (at least in some of the reported cases) does not support the multiple realizability hypothesis; and that a deeper analysis of these cases supports reductive identity physicalism in the sense that the neuroplasticity of the regions and processes in the brain that acquire the new functions do so in virtue of preexisting physical (i.e., neurological, biochemical or physical) macroscopic features and mechanisms that are shared with the physical state prior to undergoing neuroplastic processes.

Putnam (1975) found it dubious that single mental states belonging to the same kind (of type) (pain, for example) could be brought about by the firing of $\mathrm{C}$ fibers, or any other kind of brain state or neural activity. He claimed that the "brain state hypothesis" includes equating all mental states with brain states, and this is why it is implausible: "If we can find even one psychological predicate which can be applied to both a mammal and an octopus, but whose physical-chemical 'correlate' is different in the two cases, the brain-state theory has collapsed. It seems to me overwhelmingly probable that we can do this" (Putnam 1975, 436).

Putnam's multiple realizability argument is formulated in a commonly accepted structure as follows: Mental kinds are multiply realizable by distinct physical kinds. If mental kinds are multiply realizable (Bickle 2020), psychology cannot be reduced to a physical science; Therefore, psychology cannot be reduced to a physical science. 
But is it, in fact, commonplace that mental phenomena concerning neuroplasticity are multiply realized $?^{2}$ This is a question that must be taken up very carefully, both from the philosophical and the empirical perspectives. One must employ a method of testing whether the empirical evidence in neuroplasticity affirms a form of multiple realization. And of course, to do that, one must understand precisely what the multiple-realization hypotheses says. We define this hypothesis, following Putnam (1975), Fodor (1974, 1997), Davidson (1970), and others below.

Comprehensive and influential scaffolds for testing the multiple realizability hypothesis was put forth by, e.g., Bickle (2010), and more recently by Polger and Shapiro (2016). ${ }^{3}$ According to Polger and Shapiro (2016), for something to be multiply realized, it must be simultaneously the same, yet different, on two different levels. Concerning the mental and the physical, for multiple realizability to uphold, sameness is required at the level of the mental, concurrent with a difference at the physical level. Furthermore, not all differences at the physical level suffice for multiple realizability; the difference must be such that it is "relevant to their performing the same function...the differences among would-be realizers must be 'other' than

${ }^{2}$ Some authors have recently argued for other forms of multiple realizability in biology, see (Fang 2020) who argues that there is multiple realizability with relation to design principles, and (Ross 2020) who deals with multiple realizability as causal complexity. Neither speak of multiple realizability in neuroplasticity nor with relation to the mental; therefore, we don't address these arguments here.

${ }^{3}$ This is built upon earlier individual work by both Polger and Shapiro, see e.g. (Shapiro 2004, 2008; Polger 2009, Polger 2011). 
mere individual difference...the variation must not merely map onto individual differences" (Polger and Shapiro 2016, 67).

It seems that multiple realizability has two features that make it so attractive (perhaps to both philosophers and scientists): It is logically compatible with the hypothesis that the mental depends (sometimes it is said to be determined) by the physical: that is, the hypothesis that mental kinds and processes supervene on physical kinds and processes. Moreover, if multiple realizability is true, it is the ultimate reason for why mental kinds are irreducible to physical kinds (as we saw above in the quote from Sober 1999). Indeed, the conjunction of these two hypotheses paved the way for what is known today as non-reductive physicalism (including functionalism of all sorts), where the supervenience is taken to guarantee the physical basis of the mind while the irreducibility is guaranteed by the multiple realizability hypothesis (for the role played by these two hypotheses in physicalism, see Stoljar 2017; in functionalism in general, see Levin 2018; in the computational theory of mind, see Rescorla 2020). Hence the importance of the latter hypothesis for non-reductive physicalism: if the hypothesis of multiple realizability breaks down, the 'non-reductive' nature of these approaches breaks down with it. And if multiple realization can be shown to hold empirically, say in neuroplasticity, then obviously, the door is closed on identity theory (as argued by Putnam in 1975), so that neuroplasticity may be a winning case for non-reductive approaches.

What does the multiple realization hypothesis say? Following (Putnam 1975; Fodor 1974, 1997), we define multiple realizability as a generalization of the following schematic example (adapted from Putnam 1975): A psychological (or mental) kind (like pain) can be realized by many distinct physical (or biochemical) kinds: e.g., brain states of type Mam in the 
case of earthly mammals, of type Rep in the case of reptiles, of type Mol in the case of Mollusca (e.g., Octopus), etc. (see also Bickle 2020; Hemmo and Shenker 2015, 2019, 2020).

What is meant by distinct types of states here? The crucial point, in the words of Fodor (1974, 103), is this: "I am willing to believe that physics is general in the sense that it implies that any event which consists of a monetary exchange (hence any event which falls under Gresham's law) has a true description in the vocabulary of physics and in virtue of which it falls under the laws of physics. But banal considerations suggest that a description which covers all such events must be wildly disjunctive"

This means that the possible realizers of the high-level kind do not share any relevant physical (or biochemical) feature in virtue of which they realize the same high-level kind. This is Fodor's idea of a wild disjunction. If the realizers do share some physical property, it means that in all possible cases of realization, there is a strict type-type correlation between the high-level kind and the shared physical kind (or property), in which case it is a short way towards a typetype identity theory, at least insofar as empirical reasoning is concerned ${ }^{4}$, and since supervenience is assumed tout court. Our task in this paper is to show, contrary to the received view, that in all known cases of neuroplasticity, the empirical evidence suggests that such typetype correlations exist, and if so, it indirectly supports a type-type identity theory.

The hypothesis of multiple realizability, as just characterized, has been recently analyzed by Hemmo and Shenker $(2015,2019 a, 2019 b, 2020)$. In his original proposal of the "functionalstate hypothesis," as opposed to the "brain-state hypothesis" (or the "physical-chemical state hypothesis"), Putnam $(1975,436)$ has already noticed that: "the functional-state hypothesis is not

\footnotetext{
${ }^{4}$ We set aside metaphysical arguments for or against the identity theory and metaphysical notions of dependence.
} 
incompatible with dualism!" Although this is certainly true, Hemmo and Shenker argue that Putnam's observation is too weak, since the multiple realization (or realizability) hypothesis is not only compatible with dualism but rather entails dualism. They prove that non-reductive kinds necessarily assume non-reductive tokens: i.e., token-dualism, which is this: any token-realizer of a multiply realized kind must have some non-physical property which makes it realize that kind. They further show that this is the case even if the multiply realizable kinds supervene on physical kinds, as usually assumed by non-reductive approaches: in this sense, supervenience is not sufficient to ensure that the world is fundamentally physical. Although we think that Hemmo and Shenker are right, we will not defend their argument here. For the purpose of this paper, it is enough to note that if they are right, it only makes the question of whether or not neuroplasticity supports the multiple realization hypothesis more significant and interesting than is usually thought: one might have here at work a case study of empirical scientific research, which may be able to decide not only between reductive and non-reductive approaches to the mental but rather between physicalism and dualism!

This paper is structured as follows. In Section 2, we discuss structural neuroplasticity, claiming that this form of neuroplasticity provides a conceptually weak case for multiple realizability. In section 3, we examine functional neuroplasticity, theoretically a stronger case for multiple realizability. We enlist research from modern neuroscience to show that the case studies of functional neuroplasticity surveyed do not represent cases of multiple realizability. We argue that in light of the failure of both forms of neuroplasticity to provide empirical support for the multiple realizability thesis, the previously discussed types of neuroplasticity can empirically strengthen the opposite reductive theory, that of strict type-identity. In section 4 , we assert that the intuitions underlying multiple realizability derive from a graining discrepancy. We further 
argue that an identity theory recently proposed by Hemmo and Shenker's $(2015,2019,2020$;

Shenker 2017) called Flat Physicalism levels the playing field, explaining away the semblance of multiple realizability while providing a purely reductive account of the mental that is better supported by neuroscience and empirical research.

2. The Weak Argument for Multiple Realizability. As aforementioned, the multiple realizability thesis is "widely considered the most damaging objection to theories of Mind-Brain Type Identity" (Schneider 2018). We wish to investigate known cases of neuroplasticity in greater detail to determine precisely whether or not they provide evidence for instances of multiple realization. Our analysis shows that despite its intuitive ring, some forms of neuroplasticity turn out to be irrelevant to multiple realization, in the sense that they do not exhibit realization of the same mental kind by distinct physical kinds, as the hypothesis of multiple realization requires. Other forms of neuroplasticity, although relevant in the above sense, do not, in fact, support the multiple realization hypothesis.

In recent years the conventional view of neuroplasticity consists of a broad-spectrum or an umbrella topic, covering many different cases of changes that occur in the brain in response to experience or external stimulus. But neuroplasticity is not one distinct phenomenon. To understand it, especially in the context of multiple realization, it is paramount, first and foremost, to distinguish between the two principal subdivisions of neuroplasticity: structural and functional neuroplasticity,

Structural neuroplasticity is defined as "volumetric changes in discrete brain regions and the formation of new neural pathways, brought about either by the formation of new nerve fiber branches and synapses or by the growth and addition of new cells" (Costandi 2016, 16). The 
primary representative of structural neuroplasticity in the literature on the multiple realization thesis is synaptic plasticity. Synaptic plasticity refers to changes that take place in synapse strength - strengthening or weakening - between neurons. This type of neuroplasticity is thought to lie at the basis of learning mechanisms and memory. It is this classic form of plasticity, which Donald Hebb refers to in the famous quote (though he quite possibly never indeed stated it in this way) "neurons that fire together, wire together" (Demarin and Morović 2014).

Synaptic plasticity transpires during synapse formation and neurodevelopment, but for testing for multiple realizability, it is specifically necessary to explore experience-dependent synaptic plasticity of existing synapses. Research shows that the fundamental mechanisms underlying adult synaptic plasticity have a high similarity to the development of synaptic plasticity that takes place during the initial development of the brain. In what follows, we shall present state of the art on this topic as described by Bear, Connors, and Paradiso (2007). They refine synaptic modification to two processes, both based on principles of synaptic correlation.

1. When the presynaptic axon is active and, at the same time, when the postsynaptic neuron is strongly activated under the influence of other inputs, then the synapse formed by the presynaptic axon is strengthened. This is another way of stating Hebb's hypothesis, mentioned above: neurons that fire together wire together.

2. When the presynaptic axon is active and, at the same time, when the postsynaptic neuron is weakly activated by other inputs, then the synapse formed by the presynaptic axon is weakened. In other words, neurons that fire out of sync lose their link (see Bear et al. 2007). 
The neurotransmitter involved in synaptic plasticity is glutamate, which is met by two kinds of glutamate-gated ion channels in the postsynaptic cell, that act as postsynaptic receptors. These receptors are divided into two types, N-Methyl-D-aspartate (NMDA) receptors and $\alpha-$ amino-3-hydroxy-5-methyl-4-isoxazolepropionic acid (AMPA) receptors, which are located at the postsynaptic membrane. The NMDA receptor is voltage-gated with entry into the receptor blocked by $\mathrm{Mg} 2+$ ions, which must be displaced by membrane depolarization. In addition, the NMDA receptor's channel conducts Ca2+ ions (Bear et al. 2007). These differences have ramifications for the different functions of the NMDA and the AMPA receptors. For the Mg2+ block to be lifted, there must be a simultaneous presynaptic release of glutamate and postsynaptic membrane depolarization. When this occurs, the inward flow of $\mathrm{Ca} 2+$ ions can directly indicate the extent of the synchronous presynaptic and postsynaptic activation (Bear et al. 2007).

At the initial formation of a glutamatergic synapse, only NMDA receptors are present in the postsynaptic membrane. The AMPA receptors appear at a later stage of development. It is suggested that NMDA receptors serve to identify concurrent pre and postsynaptic activity. As a result of the NMDA activation (and subsequent flow of $\mathrm{Ca} 2+$ ions), the postsynaptic cell undergoes “AMPAfication” (Bear et al. 2007, 718). the appearance of AMPA receptors in the postsynaptic membrane (which were previously absent). The appearance of AMPA receptors enhances synaptic transmission. The process mentioned above leads to what is known as long term potentiation (LTP), an overall reinforcement of synaptic strength (Bear et al. 2007).

On the other hand, pre and postsynaptic firing that does not occur in synchrony leads to dissociation. The result of non-synchronous firing is weak NMDA receptor activation, which leads to a low-level influx of $\mathrm{Ca} 2+$, which in turn triggers the antithesis of LTP, known as long- 
term depression (LTD). LTD brings about a reduction in synaptic strength (resulting from a decrease in AMPA receptors) (for further details, see Bear et al. 2007).

Synaptic plasticity is taken to provide empirical support of the multiple realizability hypothesis. Following Putnam's (1975) and Fodor's $(1974,1997)$ formulation of the multiple realizability hypothesis, we reiterate that for synaptic plasticity to be a case of multiple realization, the same mental kinds must be realized (or realizable) by distinct and heterogeneous physical kinds that share absolutely no relevant physical properties. If all the (token-) realizers of a mental kind do share some physical property, then there is no multiple realization: this heterogeneity of the (token-) realizers in the various occasions of the mental kind is crucial for genuine multiple realization, since only in this case can the reduction to physical (or biochemical) kinds be blocked (as Putnam 1975; Fodor 1974, 1997; Davidson 1970 and many others stressed and conjectured).

Synaptic plasticity in the context of multiple realization has been discussed at length by Polger and Shapiro (2016). They argued that this form of neuroplasticity is not a case of multiple realization. Although their formulation of the hypothesis of multiple realization is slightly different from ours, it expresses the same idea, and their conclusion is: "Synaptic plasticity by itself is not what philosophers who advocate for multiple realization usually have in mind, for it is not an example of same-but-different. Instead, synaptic plasticity is an example of differentand-different-changes in the synaptic structure." (Polger and Shapiro 2016, 90)

To clarify the issue of what counts as multiple realization, Figdor (2010) raises the idea of 'degeneracy,' borrowed from cognitive neuroanatomy. Degeneracy was discussed at length by Polger and Shapiro as follows: "She [Figdor] allows, for example, that 'duplicate anatomical areas subserving the same function, whether or not they function redundantly, would no more 
count as [multiple realizations] than the kidneys, which are both anatomically and functionally redundant' (Figdor 2010). Figdor also notes that some neuroscientists who hypothesize widespread degeneracy do not distinguish relevant from irrelevant differences, supposing that 'a single difference in connectivity suffices for a distinct structure' (2010). At best, some kinds of degeneracy would count as examples of multiple realization” (Polger and Shapiro 2016, 134).

Figdor's degeneracy hypothesis is weaker than that which underlies the Putnam-Fodor desideratum. Only some cases of degeneracy represent cases of multiple realizability, while others do not. Moreover, Polger and Shapiro (2016) argue that Figdor's hypothesis does not rule out identity theory, but rather can be viewed as compatible with it, a conclusion that we agree with (see section 4).

The above conclusion is further supported by the brief account brought here of the biological mechanisms underlying synaptic plasticity. Synaptic plasticity results in different mental states so far as there are different neuro-biochemical processes. It is correct that something different and new occurs at the neuro-biochemical level, in learning, for example, namely the enhancement in synaptic strength and transmission brought about by the appearance of AMPA receptors, which is triggered by the activation of the NMDA receptors; but in this case, something drastically different transpires at the level of the mental. Therefore, we agree with Polger and Shapiro that this does not seem to be a case demonstrating multiple realization.

3. The Hard(er) Problem of Neuroplasticity. A seemingly stronger empirical support for multiple realization is set forth by the second major category of neuroplastic processes, functional neuroplasticity. Functional neuroplasticity is defined as "changes in some physiological aspect of nerve cell function, such as the frequency of nervous impulses or the 
probability of release of a chemical signal—both of which act to make synaptic connections stronger or weaker-or changes to the degree of synchronicity among populations of cells" (Costandi 2016, 13). Thus, the narrative of functional neuroplasticity is entirely different because, at least on the first impression, there seem to be different bio-chemical kinds involved in the realization of the same high-level function; and therefore, this case cannot be cast aside indiscriminately. In order to be thorough and present our claims regarding neuroplasticity in a manner that can be generalized, we discuss representative cases of three categories of neuroplasticity as proposed by (Grafman 2000) at length (see Section 3). The fourth category (map expansion), which we shall not discuss here, has been elaborated upon by Polger (2009) and Polger and Shapiro (2016), who showed that it does not provide evidence for multiple realizability.

3.1 Homologous Area Adaptation. One form of functional neuroplasticity is homologous area adaptation. Homologous area adaptation is the transfer of control over a specific function from one area to a parallel area on the opposite hemisphere of the brain, frequently in response to damage (Grafman 2000). Homologous area adaptation is often studied concerning cases of acquired aphasia, dysfunction of the process of language comprehension, and formation. Aphasia is characterized by an inability to convey mental representations into language, and vice versa (Damasio 1992). It provides a convenient window to homologous area adaptation as it is brought about by lesions to language-related areas that have been localized to the left cerebral hemisphere.

Research has shown that significant damage to the left hemisphere, such as that leading to aphasia, can bring about compensatory mechanisms in the right homologous areas of the cerebral cortex. The right hemisphere assumes functions once controlled entirely by the left 
hemisphere that has been destroyed by the damage (Grafman 2000). Initially, such a mechanism would seem to be a case of multiple realization, since it looks as if the same mental state is brought about by different neurological mechanisms. However, a different picture is revealed upon further scrutiny.

In response to severe injury and damage, such as a massive stroke (say to the left hemisphere), a number of sequential processes take place: activation ${ }^{5}$ of the damaged left hemisphere neural networks, disinhibition of homologous areas in the opposite right hemisphere, and competitive processes between hemispheric dominance (see also de Oliveira, Marin, and Bertolucci 2013; Spironelli and Angrilli 2015). The first stage of recovery, occurring immediately after injury, is known as the acute phase (Kiran 2012). During this phase, in which the diagnosis of aphasia is made, there is nearly no activation of either hemisphere in response to language-related tasks (Hamilton, Chrysikou, and Coslett 2011).

The next phase is the subacute stage, which lasts up to 6 months following the stroke (Kiran 2012). During this stage, what is classically referred to as plasticity takes place. It is at this stage that the right hemisphere shows activation in correlation with language-related tasks

\footnotetext{
${ }^{5}$ It warrants mention that brain imaging techniques used to measure neural activity such as fMRI are indirect approximations (they measure general neural activity indirectly by way of blood flow in the brain, not the direct activity of individual neurons). These present limitations on the extent to which multiple realization can be tested in these case studies, but obviously the limitations hold for both sides of the debate. This is an extensive far reaching discussion, which is beyond the scope of the present paper.
} 
(Hamilton et al. 2011). An influential paper by Saur et al. (2006) provides the context for the events that transpire as follows: "recovery of language occurs in a preexisting, bilateral network with an upregulation of undamaged areas and recruitment of perilesional tissue and homologue right language areas (Saur et al. 2006, 1371)." The following image further strengthens and elucidates the nature of the network:

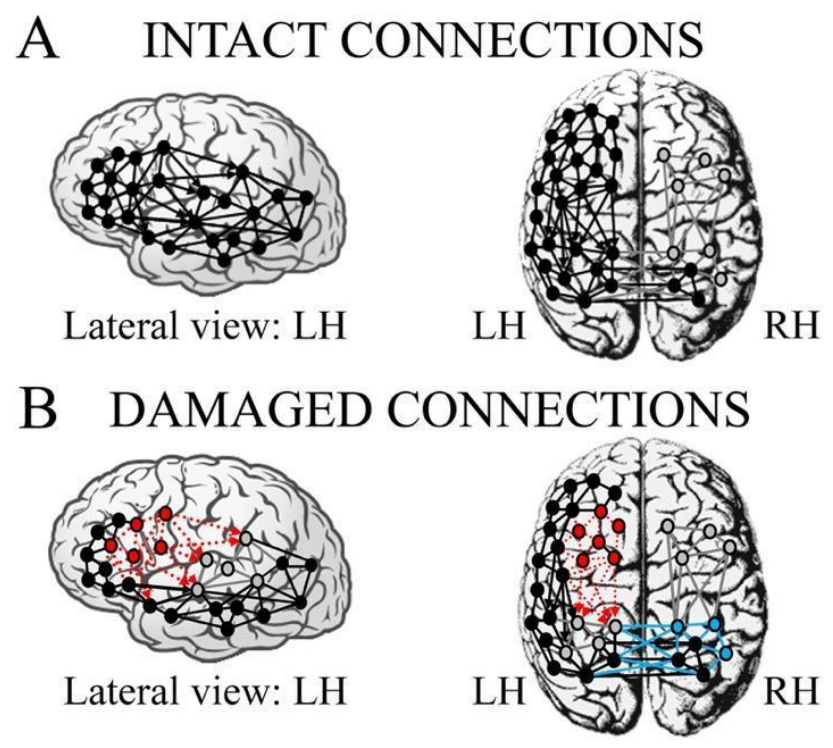

Figure 1 (from Spironelli and Angrilli 2015): Intra- and inter-hemispheric connections.

In Figure 1 (Spironelli and Angrilli 2015, 8) (A) represents a control subject. The black dots and lines depict active neural factors and connections, while the gray ones depict neural factors and connections which are inhibited by the dominant functioning areas of the left hemisphere. The areas of the right hemisphere in A show almost no activation in response to language tasks in the healthy brain. (B) represents an individual with aphasia. The red dots and connections depict the language areas, which have been damaged by stroke and are nonfunctioning. The gray dots and connections in the post-stroke left hemisphere represent areas that have been inactivated by the damaged areas adjacent to them. The blue connections and dots 
depict areas now active as a result of disinhibition and unmasking of preexisting connections that were silenced before the injury.

Once again, we reiterate, to strengthen Putnam and Fodor's idea of multiple realizability, that homologous area adaptation must exhibit a case in which mental kinds are multiply realized by distinct physical kinds with no (relevant) shared physical properties. During the subacute phase, language processes that once took place in the left hemisphere are located in the right hemisphere, so there seems to be a sense in which the language processes represent the same kind of mental state. This in itself, however, is a gross exaggeration, as the recovery of language function with relation to the homologous right hemisphere is limited at best (Hamilton et al. 2011). But more importantly, the pre-injury stage and the subacute phase are not characterized by different kinds of neuro-biochemical mechanisms. The language network is preexisting, including all relevant intra- and inter-hemispheric connections and pathways. In particular, the right hemisphere activation results from disinhibition and unmasking of connections, which is a preexisting mechanism present in both the pre-injury stage (inhibition active) and the subacute stage (inhibition inactive). Therefore, it seems to us that homologous area adaptation does not provide an exemplification of multiple realization.

3.2 Compensatory Masquerade. The recovery from major brain insult can be taken a step further with relation to multiple realizability. The final stage of recovery is known as the chronic stage, which begins around six months post-injury and can remain constant for the remainder of the person's life (Kiran 2012). During the chronic stage, the brain processes begin to normalize, and the functioning on language-related tasks begins to improve or stabilize. The scope of improvement and stabilization are directly correlated with and dependent upon the level of functional reestablishment in the left hemisphere alongside a corresponding deactivation of the 
right hemisphere. Failure in renewing left-hemispheric activation and the perpetuation of right hemisphere dominance in the chronic phase is considered by some to be maladaptive, a hindrance to the recovery of language function (Saur et al. 2006; Hamilton et al. 2011).

The reestablishment of function by the left hemisphere during the chronic phase of recovery from stroke or injury has been attributed to another form of functional neuroplasticity called compensatory masquerade. This is the reorganization of existing networks in a way that bypasses damage by establishing a substitute path to performing the same function (see FischerBaum, Jang, and Kajander 2017).

Compensatory masquerade is related to the restored and perilesional activity seen during the chronic stage of recovery. It turns out that the topography of the perilesional activity seen in recovery from left hemisphere injury and activity seen in healthy control subjects (in left inferior frontal gyrus) are similar. This similarity is taken to indicate that the compensatory perilesional activity "does not reflect the remapping of processes to nearby cortical representations. Perilesional responses more likely represent sparing of normal activity or restoration of normal activity in tissue that were not anatomically damaged by the lesion.” (Rosen et al. 2000, 1893) We take this to mean that the compensatory mechanism does not involve the reorganization of function into new areas of the left hemisphere. Instead, the left hemisphere activation is attributed to areas spared during the initial injury, or reactivation of areas that had been affected by the injury and had reassumed their prior function.

This conclusion is further strengthened by findings that show significant recovery of language function is seen mainly when certain areas of the left hemisphere, specifically in the temporal lobe, are spared and can resume their pre-lesion functions. Lesions of broad scope, 
involving the whole range of left hemisphere temporal lobe language areas, are attributed to an irreversible, significant deficit in language function (Heiss, Kessler, Thiel, Ghaemi, and Karbe 1999).

To support the hypothesis of multiple realizability in compensatory masquerade, one should show in these cases that the compensatory functions are realized by neurological processes that do not share (relevant) neuro-biochemical features with the original processes. In the chronic stage described above, language function is assumed once again by the left hemisphere. The same mental state characterizes the preinjury stage and the chronic stage (as far as there is recovery), and the same kind of neuro-biochemical state (spared areas and those that have re-attained function). Whatever capacity is not regained is the direct result of the scope of the injury, which can be attributed to a difference in the neuro-biochemical processes, but these lead to a difference in mental state. To put it plainly, whatever sameness is seen in the mental state during the chronic stage of recovery as compared to preinjury is the direct result of sameness in neuro-biochemical mechanisms, and whatever difference is seen in mental state is the result of a difference in neuro-biochemical mechanisms. The conclusion is that compensatory masquerade supports the idea of supervenience of mental states on brain states, but do not seem to support the multiple realizability thesis.

3.3 Cross-Modal Plasticity. Another form of neuroplasticity that on the face of it is taken to constitute "hard proof" of multiple realizability is known as cross-modal plasticity, a form of which is known as sensory substitution. The following quote presents this distinctive form of neuroplasticity and highlights the way it is understood: 
A most dramatic form of functional plasticity, known as sensory substitution, indicates that a cortical region previously devoted to accepting the sensory input of one modality (e.g., vision) is now capable of processing a new kind of sensory input (e.g., tactile information). (Levin and Grafman 2000, preface)

Prima facie, this form of neuroplasticity would seem to uphold, almost by definition, multiple realizability's requirement for the same kind of mental state (vision) upheld by varied modalities of input (visual and tactile).

Cross-modal neuroplasticity can be seen in braille reading blind individuals. An ample amount of research shows that tactile tasks such as braille reading activate the primary visual cortex, which is activated in sighted individuals when visualizing (Sadato et al. 1996; Grafman 2000). Seeing individuals get visual information about the world using their eyes, where the light which arrives at the retina from the outside world causes the transfer of signals to the optic nerves, which continue their journey to the lateral geniculate nucleus of the thalamus, ending up in the occipital cortex where the primary visual cortex is located (Amedi, Merabet, Bermpohl, and Pascual-Leone 2005).

In blind individuals, this process does not occur, and instead, they acquire information about the world in alternative somatosensory methods, among them braille reading. Braille reading as a cognitive mechanism involves numerous forms of processing, among them the tactile perception of the braille dots, pattern recognition, and lexical/semantic processing, and is therefore considered a tactile discrimination activity (Sadato 2005; Sadato et al. 1996). While tactile discrimination activities, such as braille reading, showed visual cortex activation in the blind, the same activities showed deactivation of the visual cortex in seeing controls (Sadato 
2005; Sadato et al. 1996). Simple tactile tasks such as sweeping a finger over Braille dots did not lead to activation of the visual cortex in neither sighted nor blind individuals (Sadato et al. 1996).

To further explore this phenomenon and its implications, research was carried out on blindfolded (seeing) individuals. It was observed that after several days, they exhibited the same visual cortex activation as blind subjects when performing tactile discrimination tasks such as braille reading (Amedi et al. 2005). The visual cortex response to tactile stimuli terminates almost immediately when the blindfold is removed, and sight is restored to the subjects. The overwhelming speed of these changes in brain activation indicates that connections between the somatosensory cortex and the visual cortex precede the external manipulation. "The speed of these changes is such that establishment of new connections is not possible" (Pascual-Leone and Hamilton 2001, 14) see also (Pascual-Leone, Amedi, Fregni and Merabet 2005). It is therefore suggested that once again, the change is the result of the unmasking of connections or "strengthening of an existing connectivity" (Bola et al. 2017) see also (Amedi et al. 2005).

These findings indicate that the occipital lobe, and the primary visual cortex, are inherently multimodal, in both seeing and blind subjects. The primary visual cortex can be recruited for visual imagery, as well as tactile and spatial imagery in a competitive manner (Pascual-Leone and Hamilton 2001).

Cross-modal neuroplasticity presents an interesting quandary for supporters of the multiple realizability hypothesis. One must determine whether, in light of research, one is willing to accept that visual cortex activation just is the physical correlate of the mental state of vision. It is certainly unacceptable to advocates of the multiple realizability hypothesis to equate specific neural correlates with vision, as this surmounts in principle to no more than equating " $\mathrm{C}$ fiber 
firing" with pain, which is the underlying idea of an identity theory. If so, it must be conceded that with relation to braille reading, the claim regarding sameness at the level of the mental fails.

If one accepts that blind braille readers and seeing readers experience the same kind of vision qualia, then the argument for multiple realization falls. In this case, the vision induced by braille reading in blind subjects and seeing subjects ultimately has the same underlying neurobiochemical mechanism, leading to visual cortex activation. Notice that if the identification of the experience of blind readers with 'vision' is rejected, this will not support the multiple realization hypothesis, but will rather give rise to a violation of the hypothesis of supervenience of the mental on the physical (since in this case, we shall have distinct kinds of experience, 'vision' and 'blind-vision' (as it were) strictly correlated with the same visual cortex activation). Thus, cross-modal plasticity seems to support an identity theory, rather than the multiple realizability hypothesis.

3.4. Sensory Substitution. Nagel (1974) explored the problem of accounting for subjective experience, the phenomenological aspects of consciousness, with his thought experiment on what it is like to be a bat. He presents the problem as follows:

Now we know that most bats... perceive the external world primarily by sonar, or echolocation, detecting the reflections, from objects within range, of their own rapid, subtly modulated, high-frequency shrieks... But bat sonar, though clearly a form of perception, is not similar in its operation to any sense that we possess, and there is no reason to suppose that it is subjectively like anything we can experience or imagine. This appears to create difficulties for the notion of what it is like to be a bat. We must consider whether any method will permit 
us to extrapolate to the inner life of the bat from our own case, and if not, what alternative methods there may be for understanding the notion. (Nagel 1974, 438)

Sensory substitution is the utilization of one sense to replace another sense that is damaged or diminished. A small percentage of the blind know reasonably well what it is like to see like a bat, using echolocation. While bats make high frequency sounds in the ultrasonic range, experienced human echolocators employ many methods of purposefully creating the necessary sound echoes, including humming, tapping, finger-snapping, and mouth clicking produced by rapid tongue motions 3-15 ms long (Thaler and Goodale 2016). The differences in pitch between sounds created by human echolocators and those created ultrasonically by bats result in human echolocation being relatively constrained in its spatial resolution. Yet expert echolocators using this method acquire an abundance of abilities attributed to vision in the seeing. Among these abilities, the recognition of object position, shape, motion, and object identity, including size and even texture with surprising accuracy (Thaler, Arnott, and Goodale 2011). Research into the neural correlates of the visual abilities attributed to trained and expert echolocators has observed significant activation of the visual cortex while echolocating (see Thaler and Goodale 2016; Thaler et al. 2011).

In this case, the argument against multiple realizability follows the same lines as those previously introduced regarding cross-modal plasticity. The outcome of the analysis depends upon whether we wish to interpret the vision achieved by echolocators as a mental state of vision or not. Either way, cross-modal plasticity and sensory substitution do not and cannot provide empirical evidence of multiple realization. However, this raises profound questions that cannot 
be ignored. Is visual cortex activation 'vision'? This would possibly be an unpopular conclusion and yet undoubtedly one that should be explored further.

Nagel, aware of the existence of blind echolocators, called for more. He proposed the challenging idea that in order to undertake the task of bridging the gap between the objective and the subjective, we could start by developing "concepts that could be used to explain to a person blind from birth what it was like to see" (Nagel 1974, 449). Developments in the study of sensory substitution take Nagel up on this challenge with the development of sensory substitution devices (SSD), which exploit cross-modal plasticity, such as the aforementioned, to create devices that substitute for disability in one sense through other modalities. An example of such sensory substitution is the vOICe visual to auditory SSD. Blind users are extensively trained in using this device, composed of a video camera connected to a computer and headphones. The computer scans the visual field and processes the visual input it receives from the camera, converting the pixels into sounds, auditory signals known as soundscapes. These auditory signals are played back into the wearer's ears, eliciting activation of the visual cortex in the blind, allowing them to recognize, identify, and locate shapes and objects (Striem-Amit, Dakwar, Reich, and Amedi 2011).

The vOICe can produce a visual image of a very high resolution of the order of tens of thousands of pixels. The resolution is sufficiently high that proficient, early blind, or congenitally blind users can surpass the World Health Organization's blindness threshold. Under these circumstances for all procedural purposes, these users, when using the SSD, are de jure low vision sighted individuals as opposed to legally blind (Striem-Amit, Guendelman, and Amedi 2012). In this unique case of practical utilization of neuroplasticity, it is quite standard interpretation, given the behavior and self-reports of these subjects, to identify their experience 
as quite similar in kind to 'vision.' And (again) this is all that's needed (on empirical grounds) to identify (in these cases) visual cortex activation with 'vision' so that the same mental state is brought about by the same neuro-biochemical mechanism. But if so, this is not a case of multiple realization.

Finally, let us just add in passing (once again) that if one resists identifying visual cortex activation with the experience of vision in these cases, despite the empirical findings, this kind of neuroplasticity will nevertheless not support the multiple realization hypothesis, but will rather give rise to a violation of the hypothesis of supervenience of the mental on the physical (since in this case, we shall have distinct kinds of experience, 'vision' and 'blind-vision' (as it were) strictly correlated with the same visual cortex activation).

4. An Alternative Explanation. Up to now, we have shown that neuroplasticity fails to provide empirical evidence supporting the multiple realizability hypothesis. In this section, we shall argue that the empirical findings in neuroplasticity seem to support the "brain-state hypothesis." That is, the empirical findings seem to give some strong support for a reductive type-type identity theory, once the latter is construed in the right way. ${ }^{6}$

\footnotetext{
${ }^{6}$ It should be noted that in order to establish a true identity claim, a further metaphysical argument is needed to account for every possible world. However, this is a topic that warrants extensive discussion that is beyond the scope of this paper: we only aim to show here that in cases of brain plasticity there are good empirical grounds for thinking that there are mind-brain correlations which threaten the multiple-realization hypothesis.
} 
Before presenting the identity theory in question, let us just say that our conclusion seems to strengthen Bechtel and Mundale's $(1999,178)$ assertion that: "When a common grain size is insisted on, as it is in scientific practice, the plausibility of multiple realizability evaporates."; as well as Bickle's $(2003,110)$ assertion concerning memory consolidation: "[I]n real science, the mental/psychological loses its status as genuinely causal mechanistic once we know how the lower-level mechanisms work." According to Bickle, the case of memory consolidation represents a direct explanation of behavioral results by "shared molecular mechanisms [that] realize shared psychological features and processes" (Bickle 2003, 157). The premise that memory can be reduced to molecular neuroscience (as Bickle argues), taken together with the hypothesis that memory and LTP are related to synaptic neuroplasticity jointly suggest that not only is synaptic plasticity not a case of multiple realization (as shown previously), but also that its underlying mechanisms can be further reduced (at least to the molecular level).

It seems to us that the above authors are right in that once common physical ground is reached, not only does neuroplasticity not provide empirical evidence for the multiple realizability hypothesis, rather neuroplasticity strengthens the approach directed by an identity theory: i.e., look for the physical-mental correlations in the pre and post neuroplastic states in the brain regions. But in the absence of all the (relevant) details of the biochemical processes, and in some cases perhaps also of the underlying physical processes in the brain, to discover the right correlations, one must have a clear and straightforward idea of what physical kinds are (this is a point stressed by Hemmo and Shenker 2020); and for this, one needs to have a straightforward theory of physical kinds. This is where the theory called Flat Physicalism, recently proposed by Hemmo and Shenker (2015, 2019a, 2019b, 2020), is relevant to this story. Flat Physicalism is a 
generalized version of statistical mechanics ${ }^{7}$, a type-type identity theory that is designed to account for all the special sciences (not only thermodynamics), including neuroscience and psychology.

Let us now very briefly describe the direction of the account of physical kinds by Flat Physicalism. In contemporary physics, the complete physical state of a system at any point in time is labeled a microstate in classical (statistical) mechanics. This represents a configuration of the entire system consisting of the precise positions and velocities of all its particles at any given moment. This description, according to physics, is the complete description of the physical state of the system at any given moment of time, which is called the microstate of this system. Knowledge of the microstate allows us to precisely predict (and retrodict) the physical evolution of the microstate across time.

A new situation arises as the number of particles in the system being described gets larger and their evolution over time gets much more complex. These systems are described by what is called in statistical mechanics macrovariables, which are partial descriptions of the complete microstate of the system. The set of all microstates that share a macrovariable is known as a macrostate. The utilization of macrovariables and macrostates allows one to make meaningful predictions about the system, without the tedious and inconceivable task of describing the full microstate and its evolution over time. This is precisely the task of statistical mechanics, pertaining to thermodynamic systems. A popular textbook describes the need for the statistical approach as follows: "At first sight we might conclude ... that, as the number of particles

\footnotetext{
${ }^{7}$ For introduction to the foundations of statistical mechanics, see e.g., (Sklar 1993; Albert 2000; Uffink 2007; Frigg 2008; Hemmo and Shenker 2012).
} 
increases, so also must the complexity and intricacy of the properties of the mechanical system, and that no trace of regularity can be found in the behaviour of a macroscopic body. This is not so, however, ... when the number of particles is very large, new types of regularity appear" (Landau and Lifshitz 1980, 1).

A prime example of the aforementioned is the thermodynamic concept of temperature. According to Flat Physicalism, what we commonly call 'temperature' of (say) an ideal gas in equilibrium, is (strictly identical with) the mechanical property average kinetic energy of all the individual particles making up the gas. This property is a macrovariable, which means the following. First, it is given by a partial description of the actual microstate of the gas. Second, it turns out that this partial description applies to an infinite number of microstates of the gas other than the actual microstate. Third, the set of all these microstates is the macrostate corresponding to the macrovariable 'average kinetic energy,' and all the microstates in this set share the same average kinetic energy of the particles; in other words, the macrovariable is a feature (or aspect) of each and every microstate of the gas that belongs to this macrostate. In statistical mechanics, macrovariables are identical with the thermodynamic properties, such as volume, pressure, temperature, and in this sense, the latter are fully reduced to mechanical properties.

Notice that there are infinitely many macrovariables other than the thermodynamic ones, which, as of now, do not play a role in the natural sciences. We stress again that the mechanical properties denoted by macrovariables are properties of the actual microstate of the system of interest, which is all there is, according to physics. By contrast, the macrostates-sets, which include counterfactual microstates and are in frequent use in statistical mechanics, are the basis of the construction of probabilities, which are about the behavior of the actual microstate of the universe and the way in which its macrovariables change over time. This is why, according to 
Hemmo and Shenker $(2012 ; 2016 ; 2019 \mathrm{ab})$, the picture of the universe given by statistical mechanics is a flat one containing a single level of reality: the so-called 'high-level' thermodynamic kind is simply (on this picture) a mechanical macrovariable of the microstate of the gas (which is nothing but a partial description of that microstate), and as such, macroproperties and micro-properties are all at the same physical level.

Hemmo and Shenker propose to regard statistical mechanics as the paradigm theory of all the special sciences, including neuroscience and psychology. They describe micro- and macrostates with regard to thermodynamics as follows: "[I]n terms of some of its aspects (or macrovariables...) then this same description is shared by many microstates, and one cannot tell which microstate among all these possible microstates (that exhibit the same macrovariable...) is the actual one. All the microstates that share the same macrovariable... form an equivalence set relative to that macrovariable" (Hemmo and Shenker 2016, 7).

While infinitely many different (possible) microstates can underlie the same macrovariable, only some of the possible macrovariables (such as the thermodynamic ones) are perceived by us (or by our measuring devices), while others are not. That is, according to statistical mechanics, when we measure thermodynamic properties, we, in fact, measure directly the corresponding macrovariables, which, as we said above, are built into the microstate; we don't measure the entire microstate of the system (see Hemmo and Shenker 2016, 2019a, 2019b, 2020; Shenker 2017 for more details about these notions and the way they feature in statistical mechanics).

Hemmo and Shenker's Flat Physicalism argues for an interpretation of type-identity in which different tokens share a physical property that is identical to a high-level (e.g., 
thermodynamic, biological, psychological) kind. In other words, two tokens can have a physical trait in common and still be very different, even wildly and radically different in other respects. This theory can explain why processes of neuroplasticity seem to be multiply realized, since they are realized by distinct token-states of the brain (actually, temporal sequences of token-states), all of which turn out to share a macroscopic physical feature, which - in a type-type identity theory - just is the high-level mental state, and therefore the neural processes they give rise to are not multiply realized. Moreover, in all the special sciences, except psychology, one can show that even if the two (temporal sequences of) token-states do not directly share a physical aspect, the two tokens (or sequences) can both include interactions with the environment, such that in both cases the environment shares the same physical feature (despite the fact that the systems do not!). This may also explain why, if one ignores the environment, multiple realization seems to hold empirically in some of the special sciences (for details of the argument, see Hemmo and Shenker 2015, 2016, 2017, 2019a, 2019b, 2020). In psychology, however, this externalist idea of extending the tokens to include the environment cannot directly hold with respect to the typeidentity account of mental states on pain of a regress. It turns out that in an identity theory, mental states belonging to the same kind must be realized by brain processes that share the same physical (or biochemical) kind. This is interesting since it gives psychology a somewhat distinguished status in Flat Physicalism despite the latter's full-blown reductive character. We skip the details here but see the argument in Hemmo and Shenker 2020.

It can be shown that the time evolution of the macrovariables of a system will, in general, be radically different from the time evolution of the microstate of that same system. For example, in classical statistical mechanics, the time evolution of the macrovariables can be stochastic, while the time evolution of the microstate is strictly deterministic. This is the way in 
which the probabilities in classical statistical mechanics arise, and this is why they are compatible with the strict determinism of the underlying microevolution in classical mechanics. This result is sufficient to recover the seeming detachment (as it were) of the laws of the special sciences from the laws of physics, despite the fact that they are fully reducible to physics; see (Hemmo and Shenker 2012, 2016, 2019a, 2019b, 2020). ${ }^{8}$

With these ideas from physics in mind, in particular, about what physical kinds are, we now go back to discussing the case studies of neuroplasticity and their ramifications concerning multiple realization vs. type-identity. We will now argue that these case studies support not only the conclusion that instances of neuroplasticity are not cases of multiple realization, but also that they support the "brain-state hypothesis," namely that the same kinds of biochemical processes in the brain are correlated with the same kinds of mental states. This gives quite a strong support to the physicalist type-type identity theory. At the fine-grained level, for neuroplasticity to occur, there are remnants of the previous pre-changed neurological structure, as we showed in the case studies analyzed above.

In homologous area adaptation, two seemingly anatomically distinct areas of the brain carry out the same kind of high-level mental process of language formation and comprehension. However, the high-level mental process has been shown to be the result of the same underlying,

\footnotetext{
${ }^{8}$ Similar remarks hold with respect to quantum statistical mechanics, where the underlying evolution of the quantum state (which is the analog of the classical microstate) may be probabilistic, but these quantum probabilities need not coincide with the statistical mechanical probabilities, precisely because the latter describe the evolution of macro-variables, while the former the evolution of the full quantum state.
} 
preexisting neuro-biochemical process. These mechanisms may be: "The unmasking of preexisting but inactive neuronal connections... by reduction of inhibitory activity (e.g., caused by a brain lesion)" (Thiel et al. 2006, 1126). This was further expanded with relation to crossmodal neuroplasticity and sensory substitution. These forms of neuroplasticity seem to convey the impression of true instances of multiple realizability, as the same high-level mental processes of visual perception are brought about by different sensory modalities, tactile or auditory. However, as we have seen, upon further inquiry of the neurological and biochemical processes involved, neuroscience points to an existing physical process. Moreover, this process can even be utilized for practical purposes of rehabilitation! According to the research into cross-modal neuroplasticity, "This change most likely reflects a strengthening of existing connectivity between the peripheral visual cortex and somatosensory cortices" (Bola et al. 2017, 1).

Without this preexisting, shared neurological remnant (whose precise neural correlates are to be elucidated by neuroscience), the plasticity cannot take place, as can be seen in the case discussed above of compensatory masquerade. Failure to tap into the spared neurological process hinders plasticity and subsequent rehabilitation and regain of function. The fact that there is a common physical remnant in the pre and post neuroplastic states suggests that these states of the relevant region of the cortex share common macrovariables.

While two seemingly distinct systems can carry out the same high-level function (biological, psychological, cognitive, or what have you), the fact that they share physical macrovariables rules out that they are genuinely multiply realized under Hemmo and Shenker's interpretation. Instead, the high-level mental processes are nothing but dynamical evolutions of the brain's macrovariables. So, although we cannot adequately compute the microstate of the brain in its entirety, in this picture, mental processes are identical with the time evolution of the 
brain's relevant macrovariables, and as such, are part and parcel of the microscopic evolution of the brain. However: "[s]hould it turn out that as a matter of empirical fact certain mental types are multiply realized by certain physical types, then Flat Physicalism with respect to the mental will end up being wrong, and one would have to opt for ending the causal chain beyond physics. Whether or not there is empirical evidence for psychophysical multiple realization is under dispute" (Hemmo and Shenker 2017, 9).

5. Conclusion. If this paper has achieved its goal, it seems that in so far as the case studies analyzed above are general enough, the empirical evidence for psychophysical multiple realization will not come about from the phenomena of neuroplasticity. As such, other empirical evidence for multiple realizability must be explored (if there is any), should the hypothesis of multiple realizability be upheld. As is well known this hypothesis is one of the central motivations supporting non-reductive approaches to the special sciences, including explicit dualistic approaches. But in the absence of empirical evidence for multiple realization, it seems to us that metaphysical arguments in support of non-reductive and even explicit dualistic approaches are much less convincing. 


\section{REFERENCES}

Albert, D. 2000. Time and Chance. Cambridge, MA: Harvard University Press.

Amedi, A., L. B. Merabet, F. Bermpohl, and A. Pascual-Leone. 2005. "The Occipital Cortex in the Blind: Lessons about Plasticity and Vision." Current Directions in Psychological Science 14 (6): 306-311.

Bear, M. F., B. W. Connors, and M. A. Paradiso. 2007. Neuroscience (Vol. 2): Lippincott Williams and Wilkins.

Bechtel, W., and J. Mundale. 1999. "Multiple Realizability Revisited: Linking Cognitive and Neural States.” Philosophy of Science, 66 (2): 175-207.

Bickle, J. 2003. Philosophy and Neuroscience: A Ruthlessly Reductive Account (Vol. 2): Springer Science and Business Media.

—. 2010. "Has the Last Decade of Challenges to the Multiple Realization Argument Provided Aid and Comfort to Psychoneural Reductionists?” Synthese 177 (2): 247-260.

—. 2020. "Multiple Realizability", The Stanford Encyclopedia of Philosophy (Summer 2020 Edition), Edward N. Zalta (ed.), URL = $<$ https://plato.stanford.edu/archives/sum2020/entries/multiple-realizability/>.

Bola, Ł., K. Siuda-Krzywicka, M. Paplińska, E. Sumera, M. Zimmermann, K. Jednoróg, A. Marchewka, and M. Szwed, M. 2017. "Structural Reorganization of the Early Visual Cortex Following Braille Training in Sighted Adults.” Scientific Reports 7 (1): 17448 .

Costandi, M. 2016. Neuroplasticity. MIT Press.

Damasio, A. R. 1992. “Aphasia.” New England Journal of Medicine 326 (8): 531-539. 
Davidson, D. 1970. “Mental Events.” In D. Davidson. 1980. Essays on Actions and Events, pp. 207-227. University of California Press.

de Oliveira, F. F., S. d. M. C. Marin, and P. H. F. Bertolucci. 2013. "Communicating With the Non-dominant Hemisphere: Implications for Neurological Rehabilitation.” Neural Regeneration Research 8 (13): 1236.

Demarin, V., and S. Morović. 2014. "Neuroplasticity.” Periodicum Biologorum 116 (2): 209-211.

Endicott, R. P. 1993. "Species-specific Properties and More Narrow Reductive Strategies.” Erkenntnis 38 (3): 303-321.

Fang, W. 2020. "Multiple Realization in Systems Biology.” Philosophy of Science 87(4): $663-684$

Figdor, C. 2010. "Neuroscience and the Multiple Realization of Cognitive Functions." Philosophy of Science 77 (3): 419-456.

Fischer-Baum, S., A. Jang, and D. Kajander. 2017. "The Cognitive Neuroplasticity of Reading Recovery Following Chronic Stroke: A Representational Similarity Analysis Approach.” Neural plasticity 2017.

Fodor, J. A. 1974. "Special Sciences (or: The Disunity of Science as a Working Hypothesis)." Synthese 28 (2): 97-115.

—_. 1997 "Special Sciences: Still Autonomous After All These Years," Nous 31, $149-163$.

Frigg, R. 2008. "A Field Guide to Recent Work on the Foundations of Statistical Mechanics," in D. Rickles (ed.), The Ashgate Companion to Contemporary Philosophy of Physics, pp. 99-196, London: Ashgate. 
Grafman, J. 2000. “Conceptualizing Functional Neuroplasticity.” Journal of Communication Disorders 33 (4): 345-356.

Hamilton, R. H., E. G. Chrysikou, and B. Coslett. 2011. "Mechanisms of Aphasia Recovery After Stroke and the Role of Noninvasive Brain Stimulation.” Brain and Language 118 (1-2): 40-50.

Heiss, W. D., J. Kessler, A. Thiel, M. Ghaemi, and H. Karbe. 1999. "Differential Capacity of Left and Right Hemispheric Areas for Compensation of Poststroke Aphasia." Annals of Neurology: Official Journal of the American Neurological Association and the Child Neurology Society 45 (4): 430-438.

Hemmo, M., and O. Shenker. 2010. Maxwell's Demon. The Journal of Philosophy 107 (8): 389-411.

—. 2012. The Road to Maxwell's Demon: Conceptual Foundations of Statistical Mechanics, Cambridge: Cambridge University Press.

—_. "The Emergence of Macroscopic Regularity." Mind \& Society 14 (2): 221-244.

—_. 2016 "Maxwell's Demon", Oxford Handbooks Online in Philosophy, Oxford University Press. doi: 10.1093/oxfordhb/9780199935314.013.63

— . 2017. "Flat Physicalism: Some Implications." Unpublished Manuscript.

— 2019a. "The Physics of Implementing Logic: Landauer's Principle and the Multiple-computations Theorem." Studies in History and Philosophy of Science Part B: Studies in History and Philosophy of Modern Physics 68: 90-105.

—. 2019b. "Two Kinds of High-Level Probability.” The Monist 102 (4): 458-477.

—. 2020. "Why Functionalism is 'Token-Dualism'.” PhiSci Archive: http://philsciarchive.pitt.edu/18073/ 
Kiran, S. 2012. "What Is the Nature of Poststroke Language Recovery and Reorganization?” ISRN Neurology 2012:786872. doi: 10.5402/2012/786872.

Landau, L. D. and E. M. Lifshitz. 1980. Statistical Physics Part 1, Course in Theoretical Physics, vol. 5. 3rd ed. Trans: J. B. Sykes and M. J. Kearsley. Oxford: Butterworth-Heinemann.

Levin, J. 2018. "Functionalism”, The Stanford Encyclopedia of Philosophy (Fall 2018 Edition), Edward N. Zalta (ed.), URL= $<$ https://plato.stanford.edu/archives/fall2018/entries/functionalism/>.

Levin, H. S., and J. Grafman. 2000. Cerebral Reorganization of Function After Brain Damage. Oxford: Oxford University Press.

Lewis, D. K. 1966. “An Argument for the Identity Theory.” The Journal of Philosophy 63 (1): 17-25.

Nagel, T. 1974. "What Is It Like to Be a Bat?" The Philosophical Review 83(4): 435-450. Pascual-Leone, A., and R. Hamilton. 2001. "The Metamodal Organization of the Brain." In C. Casanova and M. Ptito (Eds.), Progress in Brain Research, Vol. 134, pp. 427-445: Elsevier Science.

Pascual-Leone, A., A. Amedi, F. Fregni, and L. B. Merabet. 2005. "The Plastic Human Brain Cortex.” Annual Review of Neuroscience 28: 377-401.

Polger, T. W. 2009. "Evaluating the Evidence for Multiple Realization.” Synthese 167 (3): 457-472.

— . 2011. “Are Sensations Still Brain Processes?.” Philosophical Psychology 24 (1): $1-21$. 
Polger, T. W., and L. A. Shapiro. 2016. The Multiple Realization Book. Oxford: Oxford University Press.

Putnam, H. 1975. "The Nature of Mental States," in Putnam, H., Mind, Language and Reality, pp. 429-40, Cambridge: Cambridge University Press; originally published as "Psychological Predicates," in: William H. Capitan and Daniel D. Merrill (Eds.), Art, Mind and Religion, pp. 37-48, Pittsburgh, PA: University of Pittsburgh Press (1967).

Rescorla, M. 2020. “The Computational Theory of Mind”, The Stanford Encyclopedia of Philosophy (Fall 2020 Edition), Edward N. Zalta (ed.), URL = $<$ https://plato.stanford.edu/archives/fall2020/entries/computational-mind/>.

Rosen, H. J., S. E. Petersen, M. R. Linenweber, A. Z. Snyder, D. A. White, L. Chapman, A. W. Dromerick, J. A. Fiez, and M. Corbetta. 2000. "Neural Correlates of Recovery from Aphasia After Damage to Left Inferior Frontal Cortex." Neurology 55 (12): 1883-1894.

Ross, L. 2020. “Multiple Realizability from a Causal Perspective.” Philosophy of Science 87 (4): 640-662

Sadato, N. 2005. "How the Blind "See” Braille: Lessons from Functional Magnetic Resonance Imaging.” The Neuroscientist, 11 (6): 577-582.

Sadato, N., A. Pascual-Leone, J. Grafman, V. Ibañez, M. P. Deiber, G. Dold, and M. Hallett. 1996. “Activation of the Primary Visual Cortex by Braille Reading in Blind Subjects." Nature 380 (6574): 526. 
Saur, D., R. Lange, A. Baumgaertner, V. Schraknepper, K. Willmes, M. Rijntjes, and C. Weiller. 2006. "Dynamics of Language Reorganization After Stroke.” Brain 129 (6): 1371-1384.

Shapiro, L. A. 2004. The Mind Incarnate. Cambridge, MA: MIT Press.

—. 2008. "How to Test for Multiple Realization.” Philosophy of Science, 75 (5), 514-525.

Shenker, O. 2017. "Flat Physicalism: Some Consequences”. Iyyun: The Jerusalem Philosophical Quarterly 66: 211-225.

Schneider, S. 2018. "Identity Theory", Internet Encyclopedia of Philosophy, URL = $<$ http://www.iep.utm.edu/identity/>.

Sklar, L. 1993. Physics and Chance. Cambridge: Cambridge University Press.

Sober, E. 1999. “The Multiple Realizability Argument Against Reductionism.” Philosophy of Science 66 (4): 542-564.

Smart, J. J. C. 2017. “The Mind/Brain Identity Theory”, The Stanford Encyclopedia of Philosophy (Spring 2017 Edition), Edward N. Zalta (ed.), URL = $<$ https://plato.stanford.edu/archives/spr2017/entries/mind-identity/>.

Spironelli, C., and A. Angrilli. 2015. "Brain Plasticity in Aphasic Patients: Intra-and Inter-hemispheric Reorganisation of the Whole Linguistic Network Probed by N150 and N350 Components." Scientific Reports 5: 12541.

Striem-Amit, E., O. Dakwar, L. Reich, and A. Amedi. 2011. "The Large-scale Organization of 'Visual' Streams Emerges Without Visual Experience.” Cerebral Cortex 22 (7): 1698-1709. 
Striem-Amit, E., M. Guendelman, and A. Amedi. 2012. "“Visual' Acuity of The Congenitally Blind Using Visual-to-Auditory Sensory Substitution.” Plos One 7 (3): e33136.

Stoljar, D. (2017 “Physicalism”, The Stanford Encyclopedia of Philosophy (Winter 2017 Edition), Edward N. Zalta (ed.), URL = $<$ https://plato.stanford.edu/archives/win2017/entries/physicalism/>.

Thaler, L., S. R. Arnott, and M. A. Goodale. 2011. "Neural Correlates of Natural Human Echolocation in Early and Late Blind Echolocation Experts.” Plos One 6 (5): e20162.

Thaler, L., and M. A. Goodale. 2016. "Echolocation in Humans: an Overview." Wiley Interdisciplinary Reviews: Cognitive Science 7 (6): 382-393.

Thiel, A., B. Schumacher, K. Wienhard, S. Gairing, L. W. Kracht, R. Wagner, W. F. Haupt, and W. D. Heiss. 2006. "Direct Demonstration of Transcallosal Disinhibition in Language Networks." Journal of Cerebral Blood Flow \& Metabolism 26 (9): 1122-1127.

Uffink, J. 2007. “Compendium to the Foundations of Classical Statistical Physics,” in J. Butterfield and J. Earman (eds.), Handbook for the Philosophy of Physics, Part B, pp. 923-1074, Elsevier. 